\title{
RESECTION OSTEOSYNTHESIS AND BOYD AMPUTATION FOR CONGENITAL PSEUDARTHROSIS OF THE TIBIA
}

\author{
Per Edvardsen, Oslo, Norway \\ From the University of Oslo Orthopaedic Hospital, Oslo
}

True congenital pseudarthrosis of the tibia is uncommon. In most cases the discontinuity denotes the progression of a state of congenital bowing of the leg, and is either the final spontaneous fracture of increasingly weak bone during early childhood or the result of an unsuccessful attempt to correct the deformity by means of osteotomy.

Most authors agree that the weakening of the tibia is due to localised fibrous dysplasia of the diaphysis. An important point was made in 1966 by Van Nes-there is usually an associated disorder of the lower tibial growth plate. This helps to account for the very considerable total shortening by the end of growth.

In our experience the case of congenital pseudarthrosis which has a poor prognosis as regards healing is one with a sclerosed and obliterated medullary canal, a short tibia with the pseudarthrosis situated distally, and distal bowing or pseudarthrosis of the fibula as well. The osteolytic type, however, may also prove difficult to treat successfully.

If the trial operation before the age of three years fails, as often happens in this type of case, the next operation should be postponed till after the age of seven, as recommended by Lloyd-Roberts (1970). If healing is still not obtained, then in our opinion the number of further operations should be limited, firstly, because repeated operations reduce the chances of fusion; secondly, because the recurrence of a pseudarthrosis after a properly performed operation indicates a profound disturbance of osteogenesis; thirdly, because the leg in these circumstances is likely to be much shortened; and finally, because the patient deserves to be released from years of surgical care. Amputation is the final solution in such cases. As a below-knee stump in a child is apt to have complications due to skeletal growth, we decided to try a different procedure in the last case in which we failed to obtain healing of the pseudarthrosis.

\section{CASE REPORT}

The patient, a boy aged ten, was born in December 1957 with congenital bowing of the right leg showing bad prognostic criteria as regards localisation, medullary canal changes, length of the tibia and affection of the fibula (Fig. 1). By the age of two a pseudarthrosis had developed (Fig. 2) and during the next eight years the limb was operated upon nine times. The operations included medullary nailing with chip grafts, double onlay cortical grafts, double plate fixation with chips and an onlay cortical graft with heavy plate fixation. Each operation was followed within a few months by recurrence of the pseudarthrosis, even while the boy was wearing a plaster cast for protection (Fig. 3). Before the operation reported here this boy had been in hospital thirteen times by the age of nine years, with a total stay of exactly four years. Most of the time between admissions was spent either in bed at home or with his physical activity greatly restricted by protective braces or plasters. The difference of leg lengths before this operation was three centimetres, partly compensated for by overgrowth of the right femur by one centimetre.

Operation-The operation was performed in two stages. Firstly, "resection osteosynthesis" of the pseudarthrosis was carried out. The defect was exposed through the scar of a previous postero-medial incision. The vascularity of the soft tissues was found to be good. The proximal two centimetres of the distal fragment were removed with a Gigli saw, leaving the medullary 
canal open at this level. The canal was curetted and reamed out; it contained almost entirely fatty tissue down to the epiphysial plate. The distal one centimetre of the proximal fragment was then resected, at which level the medullary canal was still obliterated, and the next four centimetres were trimmed to fit snugly into the medullary canal of the distal fragment. After local resection of the fibula, the tibial fragments were impacted like the ferrule of a fishing rod to a depth of 3.5 centimetres. Further stability was secured by means of a single screw across the "ferrule". Despite the considerable shortening of the leg, the wound could be closed readily.

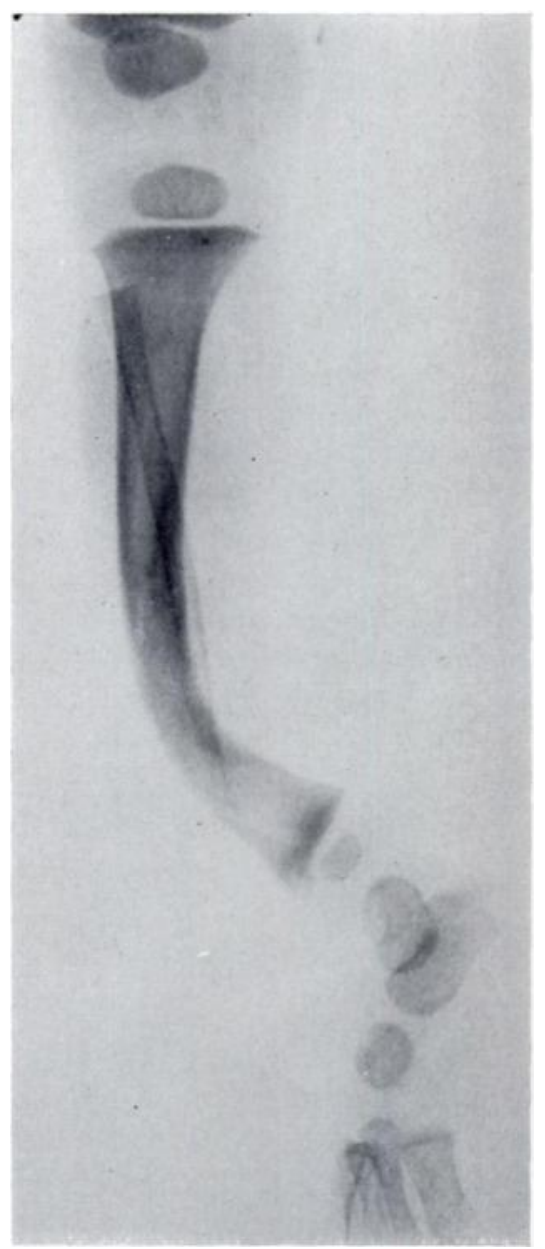

Fig. 1

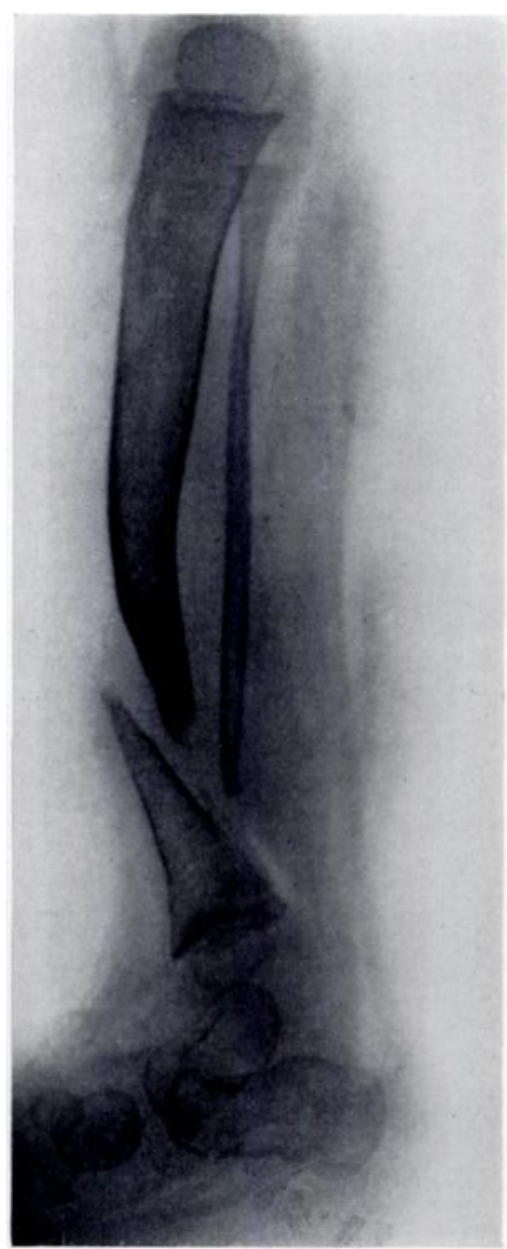

Fig. 2

Figure 1-A lateral radiograph showing congenital bowing of the tibia. Figure 2-The radiograph at two years showing pseudarthrosis of both tibia and fibula.

The post-operative course was uneventful. The osteosynthesis was protected by a plaster cast for six months, when consolidation had occurred (Fig. 4) and the second stage of the procedure was performed. This consisted of a Boyd amputation of the foot with calcaneo-tibial arthrodesis to obtain an end-bearing stump (Fig. 5). After another three months the patient was fitted with a weight-bearing leg prosthesis, which has now been used for four years without complications. The present difference in leg lengths is twelve centimetres. Serial radiographs have shown increasing thickness of the cortex and no sign of recurrence of the pseudarthrosis. 


\section{DISCUSSION}

This type of amputation was first described by Boyd in 1939; a more recent account of the procedure has been given by Tooms in 1971. We have modified the technique slightly as follows: $a$ ) the calcaneus is placed so that its inferior surface is parallel with the floor when

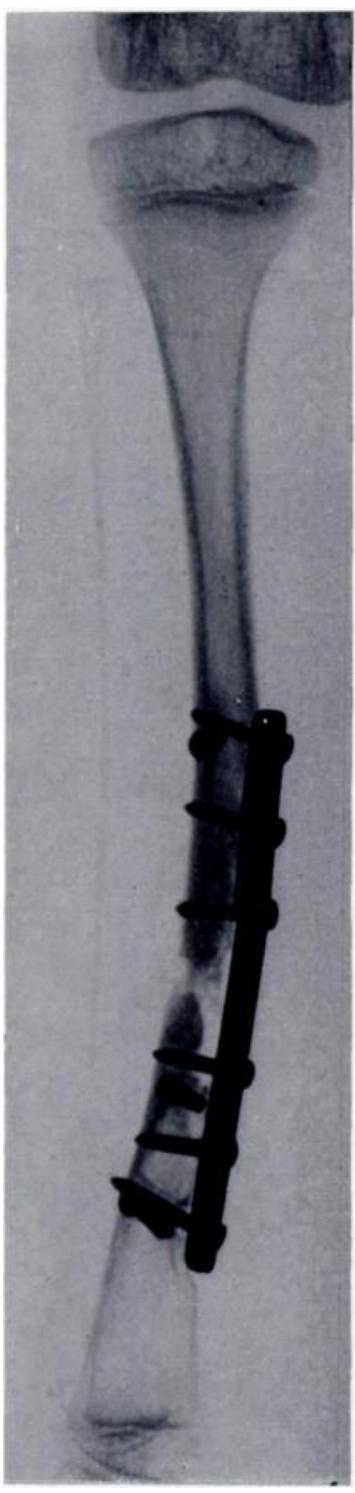

Fig. 3

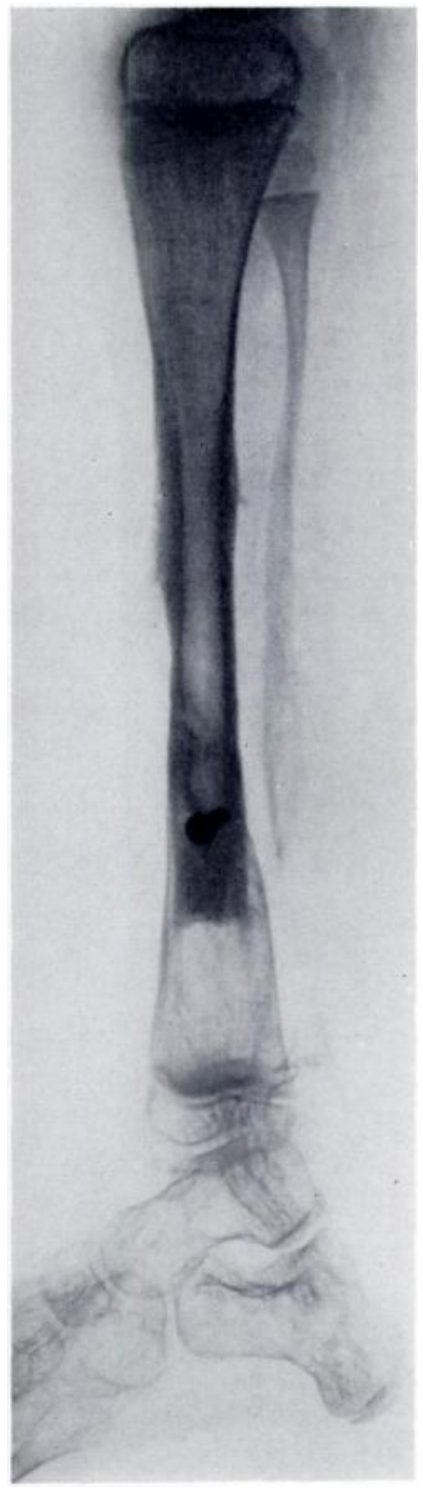

Fig. 4

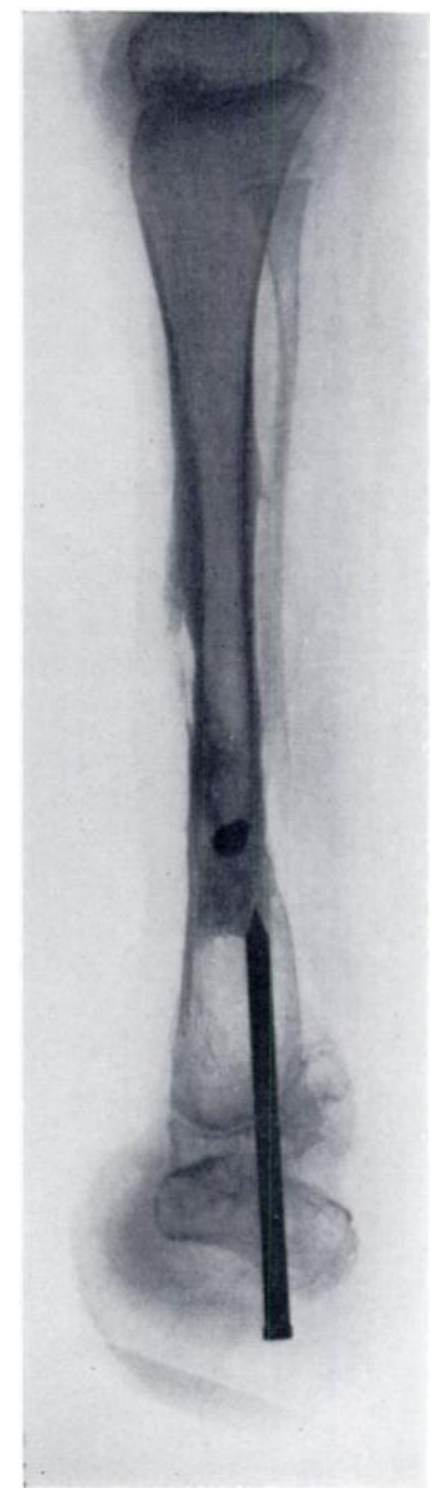

Fig. 5

Figure 3-A radiograph at 9 years showing the tibia still not united after nine operations. Figure 4-A radiograph taken six months after "resection osteosynthesis". Note the deep impaction of the upper fragment and the single screw. Figure 5-A radiograph taken after partial amputation of the foot and calcaneo-tibial arthrodesis. (The nail was driven in rather more than necessary.)

the patient is standing, in other words its weight-bearing surface is at a right angle to the tibia. $b$ ) The calcaneus is placed slightly more anteriorly than indicated by Boyd, so as to form a neat bulb underneath the leg. In this way soreness of the skin over the anterior border of the calcaneus is prevented. This detail is important in children, because the tuberosity of the calcaneus tends to elongate backwards and downwards during growth. c) The denuded joint 
surfaces of the tibia and the aligned upper surface of the calcaneus are held in position by means of a Vitallium nail driven upwards through the skin and the calcaneus into the tibia. This prevents any tilting of the calcaneus. The nail is removed after about six weeks; the screw through the "ferrule" need not be removed.

In our opinion this combination of operations has certain advantages over a conventional amputation: 1) It is based on the sound principle of removing only the dysplastic bone and allows more or less normal bone to fuse over a rather extensive area. 2) The Boyd amputation removes the torque strain on the tibia exerted by the foot, in particular when the ankle joint has decreased movement as the result of previous treatment. Thus weight-bearing can be allowed within a reasonably short time. 3) The weight-bearing stump after a Boyd amputation allows a certain amount of walking without crutches or prosthesis, for example during the night. 4) Equality of length of the lower limbs is achieved by simple adjustment of the prosthesis. 5) This type of two-stage operation may also be used for other localised affections of the tibia, such as infected post-traumatic pseudarthrosis and localised tumour.

\section{SUMMARY}

1. A case of congenital bowing of the tibia is described in which pseudarthrosis developed and recurred after nine operations performed by the age of ten years.

2. Union was finally secured by a procedure based on deep impaction of the trimmed upper fragment into the reamed-out lower fragment. This was followed by a Boyd type of partial amputation of the foot with calcaneo-tibial arthrodesis.

3. The end-bearing stump four years later is entirely satisfactory and the simple prosthesis gives equality of leg lengths.

\section{REFERENCES}

BoyD, H. B. (1939): Amputation of the Foot, with Calcaneotibial Arthrodesis. Journal of Bone and Joint Surgery, 21, 997.

LLOYD-ROBERTS, G. C. (1970): Personal communication.

TоOмs, R. E. (1971): Amputations. In Campbell's Operative Orthopaedics. Fifth edition. Volume 1, p. 852. Edited by A. H. Crenshaw. St Louis: The C. V. Mosby Company.

Van Nes, C. P. (1966): Congenital Pseudarthrosis of the Leg. Journal of Bone and Joint Surgery, 48-A, 1467. 